\title{
Real Options with Priced Regime-Switching Risk
}

\author{
John Driffill ${ }^{\mathrm{a}}$, Turalay Kenc ${ }^{\mathrm{b}}$ And Martin Sola ${ }^{\mathrm{a}, \mathrm{c}}$ \\ ${ }^{a}$ Birkbeck College, University of London \\ ${ }^{\mathrm{b}}$ University of Bradford \\ ${ }^{c}$ Universidad Torcuato Di Tella
}

This version: September 2, 2009

\begin{abstract}
We develop a model of regime-switching risk premia as well as regimedependent factor risk premia to price real options. The model incorporates the observation that the underlying risky income streams of real options are subject to discrete shifts over time as well as random changes. The presence of discrete shifts is due to systematic and unsystematic risk associated with changes in business cycles or in economic policy regimes or events such as takeovers, major changes in business plans. We analyze the impact of regimeswitching behavior on the valuation of projects and investment opportunities. We find that accounting for Markov switching risk results in a delay in the expected timing of the investment while the regime-specific factor risk premia make the possibility of a regime shift more pronounced.
\end{abstract}

Keywords: Regime-Switching Risk Premia; Regime-Dependent Risk Premia, Real Options.

JEL Classification numbers: G12; G31 


\section{Introduction}

We extend the real options valuation framework developed by McDonald and Siegel (1986) along the lines of Driffill, Raybaudi, and Sola (2003) and Guo, Miao, and Morellec (2005) to incorporate regime-dependent factor and regime switching risk premia. It is well-documented that investment falls sharply during recessions and rises sharply during booms. For example, as the US economy went into a deep recession in the early 1980's, real GNP fell 3 percent in 1981 and 1982, while investment fell by 18 percent in real terms. In the following year, as the expansion began, GNP rose 4 percent while investment rose 13 percent. Recent work models the cyclical features of firms' growth prospects in the spirit of Hamilton (1989) to capture these regime shifts in investment. For example, Guo, Miao, and Morellec (2005) demonstrated that business cycle expansion and contraction "regimes" potentially have sizable effects on the profitability or riskiness of investment and, hence, on firms' willingness to invest in physical capital. However, this recent work ignores two important channels through which business cycles affect investment decisions.

The first channel is due to the fact that the factor risk premia seem to be higher at business cycle troughs than at peaks. This phenomenon has attracted the close attention of economists. Bernanke and Kuttner (2005) highlight the importance of the perceived riskiness of stocks in the relationship between monetary policy and the stock market. Campbell and Cochrane (1999) pointed out that the risk premium for stocks will rise only to the extent that people experience declines in income and wealth that reduce their ability or willingness to absorb risk. This implies that investors would require a higher rate of return (hurdle rate) on the investment project during recessions than during expansions. The second channel is due to the fact that the

changes in risk premia over time can arise not only from regime-specific continuous (random) changes in risky income, but also from regime (discrete) shifts. It is often 
assumed that regime-switching risk is diversifiable and, hence, can be ignored when pricing primary and derivative assets. Several authors such as Naik (1993), Dai and Singleton (2003), and Wu and Zeng (2006) have stressed that regime risk premia are not only statistically significant, but also economically important for pricing purposes. We therefore develop a valuation framework that incorporates both regime-dependent risk premia and regime-switching risk premia and assess how their presence affects investment decisions.

In order to capture the effects of discrete shifts on the valuation of assets and contingent claims, researchers use either diffusion-jump [for example Merton (1976)] or switching diffusion [for example Bollen (1998)] models 1 However, applications of models with discrete shifts to real options are rare (see Schwartz and Trigeorgis (2001) for classical papers and recent contributions). Therefore our aim is to value irreversible investment opportunities under regime-switching. This extension is in the spirit of the Markov-switching model of Hamilton (1989). We then use this model to analyze the impact of the switching diffusion model on the valuation of investment projects and real options. In particular, we analyse the effects through channels of regime-switching risk premia and regime-dependent diffusion risk premia.

Related to our model of investment under uncertainty with regime shifts are Hassett and Metcalf (1999), Driffill, Raybaudi, and Sola (2003), Guo, Miao, and Morellec (2005) and Elliott, Miao, and Yu (2009). Hassett and Metcalf (1999) introduced regime shifts in investment tax credit policies within the classic single regime investment under uncertainty model of McDonald and Siegel (1986). Driffill, Raybaudi, and Sola (2003) first developed a model to price real options under switching diffusion processes. Guo, Miao, and Morellec (2005) provides a rigorous mathematical derivation of the problem examined in Driffill, Raybaudi, and Sola (2003). Finally, Elliott, Miao, and $\mathrm{Yu}$ (2009) consider regime shifts only in the cost of investment

\footnotetext{
${ }^{1}$ Naik (1993) integrates these two approaches.
} 
in the same spirit of Hassett and Metcalf (1999). But unlike Hassett and Metcalf (1999) they model the investment cost as a hidden Markov chain. Our paper can be considered as an extension to these approaches since none of these papers considers either the effects of regime-switching risk premia or regime-dependent-diffusion risk premia on investment opportunities.

We find that incorporating diffusion risk precipitates the expected time of the investment in booms and postpones the expected time of investments in recessions. We also find that when we incorporate the Markov-switching price of the risk in the model, the expected time of the investment in both regimes is delayed.

The rest of the paper is organized as follows. In section 2 we develop a valuation model of profits under a regime-switching diffusion processes. Section 3 applies this regime-switching value model to price the option to invest. Section 4 reports and discusses numerical results. Section 4 presents numerical results and investigates their implications for the project valuation and optimal timing of investment decisions across regimes. Section 5 concludes the paper.

\section{The Model}

We use a real options model of investment with regime shifts, along the lines of Driffill, Raybaudi, and Sola (2003) and Guo, Miao, and Morellec (2005) with two differences: (i) factor risk is regime-specific and (ii) regime switching is priced. Consider a firm with an infinite-horizon investment project which generates a random net cash flow (or earnings) stream of $\pi_{t}$ per unit time. The randomness in the model is not simply governed by a diffusion process with constant drift and volatility but instead characterized by an additional source of uncertainty that the dynamics of the state variable shifts between different regimes at random times. In other words, the fundamental uncertainty in the economy is generated by a Brownian motion, $\left\{W_{t}\right\}$, defined 
on a probability space $(\Omega, \mathcal{F}, P)$ and also a marked point process, more precisely a multi(two)-state Markov chain $\left\{s_{t}\right\}$. Time is continuous.

Dynamics of the state variables. The profit process $\pi$ under the actual measure $\mathbb{P}$ is formally represented by a stochastic differential equation for which we specify a regime-switching geometric Brownian motion process 2

$$
d \pi_{t}=\mu_{s_{t}} \pi_{t} d t+\sigma_{s_{t}} \pi_{t} d W_{t}
$$

The drift and diffusion terms of this profit stream are characterized by state-dependent drift $\mu_{s_{t}}$ and diffusion $\sigma_{s t}$ parameters. For the sake of tractability, it is assumed that $s_{t}$ is independent of $W_{t}$. As pointed out by Guo, Miao, and Morellec (2005), "while $\pi_{t}$ is not a Markov process, $\left(\pi_{t}, s_{t}\right)$ is jointly Makovian if at any time $t$ the state of $s_{t}$ is known."

Regime-switching. The states (regimes) $s_{t}$ of the world is modelled by a continuous time Markov chain. We suppose the firm operates in two economic regimes: 'high $\mu$ - low $\sigma$ ' and 'low $\mu$ - high $\sigma$ ', such that $s_{t}$ is a two-state Markov chain alternating between states 1 and 23 The state space of the chain $s$ can be taken to be the two unit vectors $e_{1}=(1 ; 0)^{\prime}$ and $e_{2}=(0,1)^{\prime}$ in $\mathbb{R}^{2}$. Suppose the rate matrix of the chain $s_{t}$ is $H=\left(h_{i j}\right) ; 1 \leq i, j \leq 2$, where $H$ is a $Q$-matrix and $h_{i j}>0$ for $i \neq j$,

\footnotetext{
${ }^{2} \mathrm{~A}$ candidate representation of a business cycle in a continuous-time framework might be an Ornstein-Uhlenbeck (OU) process. This adds a mean-reverting element to a standard Brownian motion process. However, in an OU process, when the state variable exceeds its mean value, its expected rate of growth is low, and when the state variable is less than its mean value, its rate of growth is relatively high. An OU process is bound to combine high levels with low expected growth, and vice versa. This restricts the way that it can represent booms and recessions.

${ }^{3}$ The two-regime Markov chain models are widely used in both macroeconomics and finance literature as it captures the observed boom-recession cycle of the real world. The early applications of these models include Bonomo and Garcia (1996) and Driffill and Sola (1998).
} 


$$
\begin{gathered}
h_{1 j}+h_{2 j}=0 \text { for } j=1,2 \text { so } h_{11}<0 \text { and } h_{22}<0 \text { : } \\
H=\left[\begin{array}{ll}
h_{11} & h_{12} \\
h_{21} & h_{22}
\end{array}\right]=\left[\begin{array}{cc}
-h_{1} & h_{2} \\
h_{1} & -h_{2}
\end{array}\right] .
\end{gathered}
$$

We will follow the Hidden Markov Model (HMM) approach4 and write the semimartingale representation of the Markov chain [see Chapter 12 of Elliot, Aggoun, and Moore (1995)] as

$$
s_{t}=s_{0}+\int_{0}^{t} H s_{v} d v+N_{t},
$$

where $N=\left\{N_{t}, \geq 0\right\}$ is an $\mathbb{R}^{2}$ valued Martingale process with respect to $\mathcal{F}_{t}^{s}$. By the result shown in Appendix B of Elliot, Aggoun, and Moore (1995) the regime $s_{t}$ has the following dynamics

$$
d s_{t}=H s_{t} d t+d N_{t}
$$

Solving the above SDE derives the following expression for the expected value of $s_{t}$

$$
E\left[s_{t}\right]=e^{H t} s_{0}
$$

where $E$ is the expectation operator taken conditional on the information available at time $t$, i.e., $E\left[. \mid \mathcal{F}_{t}\right]^{5}$ and

$$
e^{H t}=\frac{1}{c}\left(\begin{array}{cc}
h_{2} & h_{2} \\
h_{1} & h_{1}
\end{array}\right)+\frac{e^{-c t}}{c} H \quad \text { with } c=h_{1}+h_{2} .
$$

\footnotetext{
${ }^{4}$ Modelling Makov chains has been extensively studied in the literature of asset pricing under regime shifting processes. For example, Elliot, Aggoun, and Moore (1995) consider a Hidden Markov Model (HMM). Its applications to asset pricing can be found in Buffington and Elliott (2002) and Elliott, Miao, and Yu (2009). Yin and Zhang (1998) considered a Conditional Markov Chain. Its applications to the term structure can be found in Bielecki and Rutkowski (2004) and Dai and Singleton (2003). Finally, Landen (2000) uses a Marked Point Process. Its applications can be found in Wu and Zeng (2005) and Wu and Zeng (2006).

${ }^{5}$ Here the relevant information set is $\mathcal{F}_{t}^{s_{t}}$.
} 
We can obtain the probability that a transition occurs from state $e_{i}$ (say $i=1$ ) to state $e_{j}$ (say $\left.j=2\right)$ in a small time interval $(t, t+d t)$ is equal to $h_{1} d t$. Similarly, $1-h_{1} d t$ is the probability that the process remains in state $e_{i} 6$

Project valuation. Consider an investor who thinks about how much to save and consume, and what portfolio of assets to hold. Suppose the investor can buy a security whose price is $V\left(\pi_{t}, s_{t}\right)$ and that pays a dividend stream $\pi_{t}$ in state of the world $s_{t}$. The most basic pricing equation that comes from the first-order condition of that problem is the following fundamental pricing equation:

$$
V\left(\pi_{t}, s_{t}\right) u^{\prime}\left[c_{t}\left(s_{t}\right)\right]=E \int_{\tau=0}^{\infty} e^{-\rho \tau} u^{\prime}\left[c_{t+\tau}\left(s_{t+\tau}\right)\right] \pi_{t+\tau} d \tau,
$$

where $u[c]$ is the instantaneous utility function of shareholders, $c_{t}\left(s_{t}\right)$ is the consumption of the representative household at time $t$ with economy in state $s$, and $\rho$ is their subjective discount rate. As in Cochrane (2005) we define the pricing kernel (stochastic discount factor) as

$$
M\left(s_{t}\right) \equiv e^{-\rho t} u^{\prime}\left[c_{t}\left(s_{t}\right)\right]
$$

and by substituting Eq. (8) into Eq. (7) we obtain the following expression:

$$
\begin{aligned}
0 & =M\left(s_{t}\right) \pi_{t} d t+E\left[d\left(M\left(s_{t}\right) V\left(\pi_{t}, s_{t}\right)\right)\right] \\
& =M\left(s_{t}\right) \pi_{t} d t+E\left[V\left(\pi_{t}, s_{t}\right) d\left(M\left(s_{t}\right)\right]+E\left[M\left(s_{t}\right) d V\left(\pi_{t}, s_{t}\right)\right]+E\left[d\left(M\left(s_{t}\right) d V\left(\pi_{t}, s_{t}\right)\right] .\right.\right.
\end{aligned}
$$

For this regime-switching diffusion setting, following the work of Dai and Singleton (2003) the process $\left\{M\left(s_{t}\right)\right\}$ can be written as follows:

$$
d M\left(s_{t}\right)=-r^{f} M\left(s_{t}\right) d t-\lambda_{s_{t}} M\left(s_{t}\right) d Z_{t}-M\left(s_{t}\right) \Gamma_{s_{t}}\left[d s_{t}-H s_{t} d t\right],
$$

\footnotetext{
${ }^{6}$ Note that the probability $q_{i j}^{t}=\mathbb{P}\left(s_{t}=e_{j} \mid s_{0}=e_{i}\right)$ at time $t$ conditional on the initial state of the chain $s_{0}$ can be calculated as follows

$$
q_{i j}^{t}= \begin{cases}\frac{h_{j}+h_{i} e^{-c t}}{c}, & i=j \\ \frac{h_{j}-h_{i} e^{-c t}}{c}, & i \neq j .\end{cases}
$$
}


where $r^{f}$ is the risk-free rate of return, $\lambda_{s t}$ is the (regime-dependent) market price of continuous risk (diffusion risk), $\Gamma_{s t}$ is the market price of a shift from regime $s_{t}=j$ to regime $i(i \neq j ; i, j=1,2)$, and $d Z_{t}$ is the increment of a standard Wiener process, which is correlated with $d W_{t}$ with correlation coefficient $\rho_{s_{t}}$ (the correlation between returns of the market portfolio and the cash flows of the project). Note that the market price of staying in the same regime is taken to be zero. With this assumption, as explained in Footnote 11 of Dai and Singleton (2003), the market prices of risk for regime shifts cannot be determined freely. They are related in the following way:

$$
\left(1+\Gamma_{i}\right)\left(1+\Gamma_{j}\right)=1 \quad \text { for } i \neq j
$$

It is assumed that the current state of the economy is known.7

By substituting Eq. (10) into Eq. (9) and rearranging the resulting expression we obtain the following expression [see Appendix A]:

$$
r^{f} V\left(\pi_{t}, s_{t}\right) d t=\pi_{t} d t+E\left[d V\left(\pi_{t}, s_{t}\right)\right]+E\left[\frac{d M\left(s_{t}\right) d V\left(\pi_{t}, s_{t}\right)}{M\left(s_{t}\right)}\right] .
$$

This equation intuitively states that the return on the asset at the risk-free rate is equal to the flow of profits plus the expected capital gains on the asset, plus a risk premium. The capital gain on the asset in each state of the world can be written out as the sum of following three parts (the details of the derivation are provided in Appendix A):

$$
\begin{aligned}
& E\left[d V\left(\pi_{t}, e_{1}\right)\right]=\mu_{1} V_{\pi}\left(\pi_{t}, e_{1}\right) \pi d t+\frac{1}{2} \sigma_{1}^{2} V_{\pi \pi}\left(\pi_{t}, e_{1}\right) \pi^{2} d t+h_{1}\left[V\left(\pi_{t}, e_{2}\right)-V\left(\pi_{t}, e_{1}\right)\right] d t, \\
& E\left[d V\left(\pi_{t}, e_{2}\right)\right]=\mu_{2} V_{\pi}\left(\pi_{t}, e_{2}\right) \pi d t+\frac{1}{2} \sigma_{2}^{2} V_{\pi \pi}\left(\pi_{t}, e_{2}\right) \pi^{2} d t+h_{2}\left[V\left(\pi_{t}, e_{1}\right)-V\left(\pi_{t}, e_{2}\right)\right] d t .
\end{aligned}
$$

where $V_{\pi}$ and $V_{\pi \pi}$ denote, respectively, the first and second partial derivatives of $V$ with respect to $\pi$.

Similarly conditional on $s_{t}=e_{i}$ the expression for $E\left(\frac{d V d M}{M}\right)$ is written as

$$
\begin{aligned}
& E\left[\frac{d V\left(\pi_{t}, e_{1}\right) d M\left(e_{1}\right)}{M\left(e_{1}\right)}\right]=-\lambda_{1} \rho_{1} \sigma_{1} V_{\pi}\left(\pi_{t}, e_{1}\right) \pi_{t} d t-h_{1} \Gamma_{1}\left(V\left(\pi_{t}, e_{1}\right)-V\left(\pi_{t}, e_{2}\right)\right) d t, \\
& E\left[\frac{d V\left(\pi_{t}, e_{2}\right) d M\left(e_{2}\right)}{M\left(e_{2}\right)}\right]=-\lambda_{2} \rho_{2} \sigma_{2} V_{\pi}\left(\pi_{t}, e_{2}\right) \pi_{t} d t-h_{2} \Gamma_{2}\left(V\left(\pi_{t}, e_{2}\right)-V\left(\pi_{t}, e_{1}\right)\right) d t .
\end{aligned}
$$

\footnotetext{
${ }^{7}$ Veronesi (1999) and Cagetti, Hansen, Sargent, and Williams (2002) extend this specification to unobservable states.
} 
By substituting equations (12) for $d V$, (10) for $d M$ and (13) for $E[d V d M]$ in Eq. (11) and rearranging the resulting expression, we obtain:

$$
\begin{aligned}
& r^{f} V_{1}=\pi+\left[\mu_{1}-\lambda_{1} \rho_{1} \sigma_{1}\right] V_{1 \pi} \pi+\frac{1}{2} \sigma_{1}^{2} V_{1 \pi \pi} \pi^{2}+h_{1}\left(1+\Gamma_{1}\right)\left[V_{2}-V_{1}\right], \\
& r^{f} V_{2}=\pi+\left[\mu_{2}-\lambda_{2} \rho_{2} \sigma_{2}\right] V_{2 \pi} \pi+\frac{1}{2} \sigma_{2}^{2} V_{2 \pi \pi} \pi^{2}+h_{2}\left(1+\Gamma_{2}\right)\left[V_{1}-V_{2}\right],
\end{aligned}
$$

where $V_{i}=V\left(\pi_{t}, e_{i}\right)$. As pointed out by Naik (1993) and followed by Dai and Singleton (2003) in the presence of the price of regime switching risk premia the elements of the rate matrix can be written under the risk-neutral measure $\mathbb{Q}$ such as $\hat{h}_{i}=h_{i}\left(1+\Gamma_{i}\right)$ for $i=1,28$

Proposition 1. (Project Valuation) The 'fundamental' value of the project in each regime, which rules out speculative bubbles, can be obtained as

$$
V_{i}=k_{i} \pi, \quad i=e_{0}=1 \text { or } i=e_{0}=2,
$$

where $k_{i}$ is given by

$$
k_{i}=\left[r_{j}^{e}+\hat{h}_{i}-\mu_{j}\right] /\left[\prod_{i=1}^{2}\left(r_{i}^{e}+\hat{h}_{i}-\mu_{i}\right)-\prod_{i=1}^{2} \hat{h}_{i}\right] \quad \text { for } \quad i \neq j,
$$

where $r_{i}^{e}=r^{f}+\lambda_{i} \rho_{i} \sigma_{i}$

Proof. In Appendix B.

In the above equation the expected rate of return, $r_{i}^{e}$, on the project is the sum of the risk-free rate $r^{f}$ and the diffusion (factor) risk premium $\lambda_{i} \rho_{i} \sigma_{i}$. The expected returng is written in this conventional way for ease of comparison. The expression for $k$ can be written in several alternative ways. It can be expressed in terms of either the risk-free rate or the instantaneous expected rate of return $\hat{r}_{i}^{e}=r_{i}^{e}+h_{i} \Gamma_{i}$ :

$$
k_{i}=\left[r^{f}+\hat{h}_{i}-\hat{\mu}_{j}\right] /\left[\prod_{i=1}^{2}\left(r_{i}^{f}+\hat{h}_{i}-\hat{\mu}_{i}\right)-\prod_{i=1}^{2} \hat{h}_{i}\right] \quad \text { for } \quad i \neq j
$$

\footnotetext{
${ }^{8}$ It must be noted that in Dai and Singleton $(2003) h$ under $\mathbb{Q}$ is $\hat{h}_{i}=h_{i}\left(1-\Gamma_{i}\right)$ while Proposition 3 of Naik (1993) confirms our result, $\hat{h}_{i}=h_{i}\left(1+\Gamma_{i}\right)$.

${ }^{9}$ See Shackleton and Wojakowski (2002) for an analysis of the expected return of real options.
} 


$$
k_{i}=\left[\hat{r}^{e}+h_{i}-\mu_{j}\right] /\left[\prod_{i=1}^{2}\left(\hat{r}_{i}^{e}+h_{i}-\mu_{i}\right)-\prod_{i=1}^{2} \hat{h}_{i}\right] \quad \text { for } \quad i \neq j
$$

where $\hat{\mu}=\mu_{i}-\lambda_{i} \rho_{i} \sigma_{i}$ which is the drift term of the profit process under $\mathbb{Q}$.

Notice that when the two regimes $s_{t} \in S$ are switched off [i.e., $h_{1}=h_{2}=0$, $\mu_{1}=\mu_{2}$ and $\left.\hat{r}_{1}^{e}=\hat{r}_{2}^{e}\right]$, Eqs. (15a) and (15b) collapse to the value of the project under a single regime [see Dixit and Pindyck (1994) (Chapter 6)]:

$$
V=\pi /\left[r^{e}-\mu\right]
$$

Notice that for the value of the project, $V$, to be bounded, the discount value, $r^{e}$, should be greater than the drift, $\mu$. The difference $r^{e}-\mu$ (usually denoted by $\delta$ ) represents a measure of dividends. The expression $r^{e}-\mu=\delta$ can be thought as an equilibrium relationship. If some of the parameters of the model change, the equilibrium must be restored, but which of the three magnitudes adjusts depends on the underlying technology and behavior.

Remark 1. In the regime-dependent model, the presence of regime-switching risk (i.e., capital gains due to discrete shifts), implies that $\delta_{i}$ differ from $\hat{r}_{i}^{e}-\mu_{i}$ (as seen from Eq. (15)) since rational investors take regime switches into account, and therefore the dividend (or convenience) yield, $k_{i}$, is determined by the transition probabilities $h_{j}$, the regime-dependent $\hat{r}_{j}^{e}$, and $\mu_{j}, j=1,2$.

We perform a numerical analysis of the model, choosing the parameter values in line with those used in Driffill, Raybaudi, and Sola (2003), Guo, Miao, and Morellec (2005) and Dai and Singleton (2003) in order to evaluate the impact of allowing for regime-switching price of the risk on $k_{i}$. We do this assuming that expansions are characterized by a high growth rate and low volatility of profits, whereas economic recessions have a low growth rate and high volatility. The numerical results reported in Table 1 conform with the results for asset prices at the aggregate level obtained by Cecchetti, Lam, and Mark (1990) and Driffill and Sola (1998) when the required rate 
of return is not regime-specific and exclusive of the regime switching risk premium. For the chosen values presented in Table 1, the single regime model generates $k=$ 28.33 .

Table 1: The Impact of Regime Switching on Valuation

\begin{tabular}{|c|c|c|c|c|c|c|c|}
\hline & $\lambda_{i}$ & $\rho_{i}$ & $\mu_{i}$ & $\sigma_{i}$ & $r^{e}$ & $h_{i} \Gamma_{i}$ & $k_{i}$ \\
\hline \multicolumn{8}{|c|}{ Identical Diffusion Risk Premia $\left(r_{1}^{e}=r_{2}^{e}\right)$} \\
\hline Regime 1 & & & $6.50 \%$ & $7.00 \%$ & $7.43 \%$ & & 40.12 \\
\hline Regime 2 & & & $1.30 \%$ & $15.00 \%$ & $7.43 \%$ & & 30.47 \\
\hline \multicolumn{8}{|c|}{ Regime Varying Diffusion Risk Premia $\left(r_{0}^{e} \neq r_{1}^{e}\right)$} \\
\hline Regime 1 & 0.60 & 0.30 & $6.50 \%$ & $7.00 \%$ & $6.26 \%$ & & 52.37 \\
\hline Regime 2 & 0.30 & 0.80 & $1.30 \%$ & $15.00 \%$ & $8.60 \%$ & & 35.05 \\
\hline \multicolumn{8}{|c|}{ Regime Switching Risk Premia $\left(\hat{r}^{e}\right)$} \\
\hline Regime 1 & 0.60 & 0.30 & $6.50 \%$ & $7.00 \%$ & $6.26 \%$ & $0.02 \%$ & 36.66 \\
\hline Regime 2 & 0.30 & 0.80 & $1.30 \%$ & $15.00 \%$ & $8.60 \%$ & $-0.02 \%$ & 24.53 \\
\hline
\end{tabular}

$$
r^{f}=5 \%, h_{1}=0.065, h_{2}=0.090, \Gamma_{1}=0.3800 \text { and } \Gamma_{2}=-0.2754
$$

The numerical experiment of the model when the diffusion-risk premia are assumed to be invariant across regimes indicates that the value of the project in the two regimes can be substantially different: a multiplier of 30.47 in regime 2 (recession) as opposed to 40.12 in regime 1 (boom). The expansion regime (with a high growth rate and low volatility of profits) is associated with higher values of $k$ (and hence higher project values, $V$ ). Similarly, recessions (low growth rate and high volatility yield) are associated with lower values of $k$ (and hence lower values of the project).

To relax the assumption that the discount rates are the same in the two states, we choose values for $\lambda$ and $\rho$ consistent with the observation that the risk premium 
is higher during recessions than during expansions. We choose values for the market price of the risk and for the coefficient of correlation (between the pricing kernel and the project value), which are higher in regime 1 than in regime 2. Notice that having $\lambda$ and $\rho$ as regime-specific parameters implies that the risk premia are also regimedependent. Adding regime specific diffusion risk to the model further increases the divergence between the values of the project in each regime (since we discount more the future profits in recessions than in booms).

Remark 2. Relaxing the identical diffusion risk premia assumption widely used in the literature increases the difference between the value of the project in the two regimes.

The assumption of regime-specific diffusion risk premia implies expected rates of return on the projects of $r_{1}^{e}=6.26 \%$ and $r_{2}^{e}=8.60 \%$, as opposed to the single rate of return $r^{e}=7.43 \%$ associated with the assumption of identical diffusion risk premia. Similarly, the difference between the regime convenience yields, $k$, increases. Under this assumption, the boom regime $k$-value rises from the value 40.12 (obtained under the identical diffusion risk premia assumption) to 52.37 in regime 1, and increases from 30.47 to 35.05 in regime 2 (recession). These differences in $r^{e}$ and $k$ in turn increase the difference between the value of the project in the two regimes. This conforms to the finding of Abel and Blanchard (1986), who highlight the effects of variations in the cost of capital on the present value series.

Finally, we analyze the effects of pricing the regime-switching risk on the project valuation. We find the following.

Remark 3. Pricing the regime-switching risk decreases the dividend yield in both states because the projects are discounted at a higher rate.

The last two rows of Table 1 summarize the effect of pricing regime-switching on the dividend yield. We also report $h_{i} \Gamma_{i}$ which measures the risk premium in 
regime $i$ due to regime-switching. The required rates of return of the project increase, as expected, by the extent of the regime-switching risk premium to approximately $\hat{r}_{1}^{e}=6.46 \%$ and $\hat{r}_{2}^{e}=8.40 \%$. Similarly, the project's convenience yields decrease, reflecting the rise in the expected rates of return.

Remark 4. An increase (in each state) in the price of the regime-switching risk can be interpreted as an increase in the probability of switching to the recession state (from a boom) and as a reduction in the probability of switching to the boom (from a recession).

This symmetry can be easily seen in Eq. (14) in the text. Then, an increase in the probability of switching to a recession and/or a decrease in the probability of switching to a boom have the effect of reducing the value of the project.

\section{$3 \quad$ Valuing the Option to Invest}

In this section we consider regime switching and optimal investment timing in a real option framework in the spirit of McDonald and Siegel (1986). We find the value of the option to invest, $F$, and the critical profit stream, $\pi^{*}$, at which it is optimal to pay a sunk cost, $I$, for a project, $V$. The investment opportunity is assumed to last forever and therefore it is equivalent to a perpetual call option - the right but not the obligation to buy a share of stock at a prespecified price.

Since the profit process follows the two-regime-switching process defined in Eq. (1), there exists a different trigger threshold, $\pi_{i}^{*}$, for each regime $i$. That is, there are two optimal critical values, $\pi_{1}^{*}$ and $\pi_{2}^{*}$, with $\pi_{1}^{*}<\pi_{2}^{*}$, (as in Hassett and Metcalf (1999), Driffill, Raybaudi, and Sola (2003) and Guo, Miao, and Morellec (2005)) and thus there are three relevant regions in the profit space: In region $1\left(\pi \leq \pi_{1}^{*}\right)$, there is no investment regardless of the value of $\pi$ until the profits stream reaches $\pi_{1}^{*}$, where, provided that the economy is in state 1 , investment takes place. In region 2 
$\left(\pi_{1}^{*} \leq \pi \leq \pi_{2}^{*}\right)$, there is investment if the economy is in regime 1 , and in region 3 $\left(\pi_{1}^{*}<\pi_{2}^{*} \leq \pi\right)$ there is investment regardless of the state of the economy 10

The investment problem in each region is defined in Table 2, which shows the equations that characterize the investment opportunities, $F$, across regions.

Table 2: Investment Problem in Each Region

\begin{tabular}{l|l}
\hline \hline In region 1: & $\mu_{1} \pi F_{1 \pi}+\frac{1}{2} \sigma_{1}^{2} \pi_{1}^{2} F_{1 \pi \pi}+\hat{h}_{1}\left(F_{2}-F_{1}\right)-r^{f} F_{1}-\rho_{1} \lambda_{1} \sigma_{1} \pi F_{1 \pi}=0$ \\
$\left(\pi \leq \pi_{1}^{*}\right)$ & $\mu_{2} \pi F_{2 \pi}+\frac{1}{2} \sigma_{2}^{2} \pi_{2}^{2} F_{2 \pi \pi}+\hat{h}_{2}\left(F_{1}-F_{2}\right)-r^{f} F_{2}-\rho_{2} \lambda_{2} \sigma_{2} \pi F_{2 \pi}=0$ \\
\hline In region 2: & $F_{1} \leq V_{1}-I$ \\
$\left(\pi_{1}^{*} \leq \pi \leq \pi_{2}^{*}\right)$ & $\mu_{2} \pi F_{2 \pi}+\frac{1}{2} \sigma_{2}^{2} \pi_{2}^{2} F_{2 \pi \pi}+\hat{h}_{2}\left(F_{1}-F_{2}\right)-r^{f} F_{2}-\rho_{2} \lambda_{2} \sigma_{2} \pi F_{2 \pi}=0$ \\
\hline In region 3: & $F_{1} \leq V_{1}-I$ \\
$\left(\pi_{2}^{*} \leq \pi\right)$ & $F_{2} \leq V_{2}-I$ \\
\hline \hline
\end{tabular}

$\overline{\text { where } F_{i \pi} \text { and } F_{i \pi \pi} \text { denote, respectively, the first and second partial derivatives of } F}$ with respect to $\pi$ in state $i . F_{i}=F\left(\pi_{t}, e_{i}\right)$ is the value of the function in state $i$, and so on.

This system of equations can be solved subject to eight (boundary) conditions, namely

(i) zero value conditions

$$
F_{i}(0)=0, \quad i=1,2
$$

(ii) value matching conditions

$$
F_{i}\left(\pi_{i}^{*}\right)=V_{i}\left(\pi_{i}^{*}\right)-I, \quad i=1,2 ;
$$

\footnotetext{
${ }^{10}$ Because of the corresponding investment action, the sets $\left(0, \pi_{1}^{*}\right)$ and $\left(\pi_{2}^{*}, \infty\right)$ are often referred as to the inaction region and the action region, respectively. The set $\left[\pi_{1}^{*}, \pi_{2}^{*}\right]$ is called transient region (see Guo (2001)).
} 
(iii) smooth-pasting condition 11

$$
F_{i}^{\prime}\left(\pi_{i}^{*}\right)=V_{i}^{\prime}\left(\pi_{i}^{*}\right), \quad i=1,2
$$

(iv) continuity condition 12

$$
\begin{aligned}
& \lim _{\pi \downarrow \pi_{1}^{*-}} F_{2}(\pi)=\lim _{\pi \uparrow \pi_{1}^{*+}} F_{2}(\pi), \\
& \lim _{\pi \downarrow \pi_{1}^{*-}} F_{2}^{\prime}(\pi)=\lim _{\pi \uparrow \pi_{1}^{*+}} F_{2}^{\prime}(\pi) .
\end{aligned}
$$

Proposition 2. (Option Valuation) Solutions for $F_{1}$ and $F_{2}$ across regions are given by the expressions reported in Table 3:

Proof. See Appendix C.

In Appendix $\mathrm{C}$ we show that the system of six equations in Table 3 can be solved for six unknowns: $\pi_{1}^{*}, \pi_{2}^{*}, A_{1}, A_{2}, C_{1}$ and $C_{2}$. The unknowns $B_{1}$ and $B_{2}$ are obtained from a relationship between $A^{\prime} s$ and $B^{\prime} s$ (presented in Appendix $\mathrm{C}$ ): $B_{1}=l_{1} A_{1}$ and $B_{2}=l_{2} A_{2}$. The solution for the value of the option to invest in region $1,\left(0, \pi_{1}^{*}\right]$ accounts for the possibility that the investor may invest in the other regime. In this region the value of the option in each regime $i$ has two components (see Appendix $\mathrm{C}$ ) associated with the negative roots of the polynomial in $\gamma$ (see tables and Appendix C). The value of the option $F_{2}$ in the region $\left[\pi_{1}^{*}, \pi_{2}^{*}\right]$ reflects the fact that, if the economy switches from regime 2 to 1 , the firm immediately invests.

\footnotetext{
${ }^{11}$ Technically these conditions are meant to ensure that the slopes of the functions $F_{i}$ and $V_{i}-I$ are equal at the investment thresholds $\pi_{1}^{*}$ and $\pi_{2}^{*}$. Intuitively, they imply that the rate of returns to the option and the project net of the investment cost are equal at the investment thresholds.

${ }^{12}$ They ensure the smoothness of the option value function at the boundary between the inaction region and the transient region [see Driffill et al. (2003) and Guo, Miao, and Morellec (2005)].
} 
Table 3: Solution for Investment Problem in Each Region

\begin{tabular}{l|l}
\hline \hline In region 1: & $F_{1}=\sum_{i=1}^{2} A_{i} \pi^{\gamma_{i}}$ \\
$\left(\pi \leq \pi_{1}^{*}\right)$ & $F_{2}=\sum_{i=1}^{2} B_{i} \pi^{\gamma_{i}}$ \\
\hline In region 2: & $F_{1} \leq k_{1} \pi-I$ \\
$\left(\pi_{1}^{*}<\pi \leq \pi_{2}^{*}\right)$ & $F_{2}(\pi)=C_{1} \pi^{\zeta_{1}}+C_{2} \pi^{\zeta_{2}}-\frac{\hat{h}_{2} I}{\left(\hat{h}_{2}+r_{2}^{e}\right)}+\frac{\hat{h}_{2} k_{1}}{\left(\hat{h}_{2}+r_{2}^{e}\right)-\mu_{2}} \pi$ \\
\hline In region 3: & $F_{1} \leq k_{1} \pi-I$ \\
$\left(\pi_{2}^{*} \leq \pi\right)$ & $F_{2} \leq k_{2} \pi-I$ \\
\hline \hline
\end{tabular}

where $\gamma_{1}$ and $\gamma_{2}$ are distinct real roots of the quadratic equations defined in Eq. (C.4) in Appendix C; $\zeta_{1}$ and $\zeta_{2}$ are similarly distinct real roots of the quadratic equation defined in Eq. (C.9) in Appendix C; $A_{1}, A_{2}, \pi_{1}^{*}, \pi_{2}^{*}, C_{1}, C_{2}$ are constants to be determined by the model's two value matching conditions, two smoothpasting conditions and two continuity conditions as described in Appendix C. $B_{1}$ and $B_{2}$ are determined as function of $A_{1}$ and $A_{2}$.

\section{Sensitivity Analysis}

In this section we conduct a sensitivity analysis of the impact of regime-dependent market prices of diffusion risk and regime-switching risk on capital budgeting (investment) decisions. The baseline values for the key parameters are given in Table 1; the sunk cost $I$ takes a value of 50 in the baseline case. The threshold values of profits required for entry, and values of the project at entry, are calculated numerically, and listed in Table 4. Figure 1 plots the state dependent project and the option values as a function of the level of profit 13 Table 4 reports critical values of the profit streams of the project, derived under the assumption of both regime-switching risk

\footnotetext{
${ }^{13}$ Figure 1 is generated for the model with regime-varying diffusion (factor) risk premia and priced regime-switching risk premia.
} 
premia and regime-dependent market prices of diffusion risk. For comparison, we also provide values for the single regime case. As seen from Figure 1, the investment thresholds and project values in the expansion regime (i.e., regime 1) differ considerably from those in the recession regime (i.e., regime 2). These results further vary across the three models we consider, namely, (i) identical diffusion risk premia, (ii) regime varying diffusion risk premia and (iii) regime-switching risk premia.

Under the assumption of identical diffusion risk premia, we find that the critical profit level in regime 2 (recession) is 5.1 while the value in regime 1 (boom) is 2.65 . This result is due to the fact that the better market conditions associated with the boom induce the firm to invest at a lower critical value in the boom than in the recession regime. Notice that the critical value (and project value) in the single regime model is not the average of the two regimes' values. Consider the use of a single regime diffusion process when the true underlying uncertainty is subject to Markov switches in the mean and in the variance. The single regime model omits the changes in mean and therefore would typically overestimate its variance. This miscalculation would be exacerbated the greater the difference between the means of the two states. If the drifts in the two regimes are very different, then the calculated variance (which wrongly omits the changes in the mean) in the single regime diffusion process is likely to be larger than the variance in either regime of the switching diffusion model. Since the value of the option to invest depends strongly on the variance of the returns to the investment, modelling data generated by a switching diffusion process as a single regime diffusion process is likely to give highly misleading predictions about the optimal timing of investment decisions. Notice that this effect on the single regime model will be present regardless of the characterization of recession as the low and high variance state.

Remark 5. Relaxing the assumption of identical diffusion risk premia increases the critical profits level in the recessions and decreases it in the booms. 
Allowing the diffusion risk premia to vary across regimes (the risk is assumed to be low in booms and high in recessions) further lowers the critical profit level in the boom to 2.05 (from 2.65 in the case of identical diffusion risk premia) and increases the critical value to 5.51 in the recession (from 5.11 under identical diffusion risk premia). This result is due to the fact that profits are discounted more heavily in a recession than in a boom. It is in line with empirical evidence that private (investment) spending increases during expansions and drops during recessions.

\subsection{Effects of Introducing Regime-Switching Risk Premia}

Adding regime-switching risk premia to the discount rate increases the critical values that trigger investment in both regimes. Table 5 reports the results from the sensitivity analysis carried out for $\pi_{i}, k_{i}$, and $V_{i}$ with respect to $\Gamma_{i}$. The results show that the higher is the market price of the risk $\Gamma_{1}$ (i.e., the higher the probability) of switching from a boom to a recession, the greater is the critical value $\pi_{1}^{*}$. This is expected since, if there is a higher prospect of switching to a recession (or making a capital loss), the agents would only invest in a boom at a higher threshold. Also, the higher is the market price of the risk (i.e., the lower the probability) of switching from a recession to a boom, the higher is the critical value $\pi_{2}^{*}$. This reflects the fact that the reduction in the possibility of making a capital gain when changing regimes discourages investment.

Also, a high market price of the risk of switching from a boom regime to a recession regime lowers the convenience yield $k$. This reflects the fact that future profits are discounted at a higher rate. The reduction in $k_{1}$ outweighs the rise in $\pi_{1}^{*}$ and thus leads to a decrease in $V_{1}$. Notice that when we incorporate Markov-switching premia, the expected timing of the investment is postponed both in the booms and in the recessions This is due to the fact that adding this extra component to the discount factor modifies the critical thresholds but does not modify the driving process. 
Table 4: Thresholds and Project Values

\begin{tabular}{l|c|c|c}
\hline \hline & Two Regime Model & Single Regime Model \\
\hline \multicolumn{4}{c}{ Identical Diffusion Risk Premia $\left(r_{1}^{e}=r_{2}^{e}\right)$} \\
\hline Critical Value $\pi^{*}$ & $\pi_{1}^{*}=2.65$ & $\pi_{2}^{*}=5.11$ & $\pi^{*}=2.20$ \\
Project Value $V\left(\pi^{*}\right)$ & $V\left(\pi_{1}^{*}\right)=106.27$ & $V\left(\pi_{2}^{*}\right)=155.62$ & $V\left(p i^{*}\right)=62.29$ \\
\hline \multicolumn{4}{c}{ Regime Varying Diffusion Risk Premia $\left(r_{1}^{e} \neq r_{2}^{e}\right)$} \\
\hline Critical Value $\pi^{*}$ & $\pi_{0}^{*}=2.05$ & $\pi_{1}^{*}=5.51$ & $\pi^{*}=2.20$ \\
Project Value $V\left(\pi^{*}\right)$ & $V\left(\pi_{1}^{*}\right)=107.56$ & $V\left(\pi_{2}^{*}\right)=193.28$ & $V\left(\pi^{*}\right)=62.29$ \\
\hline \multicolumn{4}{c}{ Regime Switching Risk Premia $\left(r^{e}+h_{i} \Gamma_{i}\right)$} \\
\hline Critical Value $\pi^{*}$ & $\pi_{1}^{*}=2.40$ & $\pi_{2}^{*}=5.62$ & $\pi^{*}=2.20$ \\
Project Value $V\left(\pi^{*}\right)$ & $V\left(\pi_{1}^{*}\right)=88.00$ & $V\left(\pi_{2}^{*}\right)=137.89$ & $V\left(\pi^{*}\right)=62.29$ \\
\hline \hline
\end{tabular}

$\Gamma_{1}=0.3800, \Gamma_{2}=-0.2754, I=50$ and the baseline values for the rest of the parameters are given in Table 1.

\subsection{Elasticity Analysis}

We explore how changes in the parameters affect optimal investment decisions. Table 6 reports the elasticities of the five key variables in each regime $i=1$ (boom),2 (recession): namely, (i) the critical values of the profit $\left(\pi_{i}^{*}\right)$, (ii) the convenience yield $\left(k_{i}\right)$, (iii) regime-switching risk premia $\left(h_{i} \Gamma_{i}\right)$, (iv) the project value $\left(V\left(\pi_{i}^{*}\right)\right)$, and $(v)$ the value of the option $\left(F\left(\pi_{i}^{*}\right)\right)$, with respect to the mean growth rate of the profit process $\mu_{i}$, its volatility $\sigma_{i}$, the market price of diffusion risk $\lambda_{i}$, the probability of regime-switching $h_{i}$ and the market price of regime-switching risk $\Gamma_{i}$ in each regime.

The first two rows of Table [ show that all the five key variables (in each state), except the critical value of profits, respond positively to increases in the expected growth rate of profits in either state $\left(\mu_{i}, i=1,2\right)$. The reason for the negative elasticity of the critical value of profits is that an increase in the drift parameter has 
two opposite effects: (i) it increases the value of the project and (ii) it increases the value of the option to invest. The first effect dominates and thus a higher drift results in a lower entry threshold. Also, a change in the drift parameter affects the value of the option in two different ways: on the one hand, a change in profits affects negatively the value of the option to invest (it is like an increase in dividends reducing the value of a call), while on the other hand, since a change in the drift increases the value of the project, it also increases the value of the option (it is like an increase in the value of the underlying asset increasing the value of a call). In our numerical analysis the second effect was dominant.

The next two (third and fourth) rows of Table 6 show the elasticities with respect to $\lambda_{i}$ (changes in $\lambda_{i}$ correspond to changes in the discount rates). All the five key variables ( in each state), except the critical level of profits, have a negative elasticity. The reason for the positive elasticity of the critical value of profits is that an increase in the discount rate has two opposite effects. As $\lambda_{i}$ increases, the critical project-value decreases (since an increase in $\lambda$ implies that future profits are discounted at higher rate) and also the value of the option to invest diminishes. The first effect dominates so the critical profits are higher.

The fifth and sixth rows of Table 6 report the effects of changes in the variances of the profit process $\left(\sigma_{i}, i=1,2\right)$. The effects on the relevant critical thresholds are complex since this parameter affects not only the driving process but also the discount rate. If we observe the effect on the critical level of profits we see that the range of inaction increases (a higher critical level to carry out the investment) in both cases and in both states. Although this outcome is in line with results obtained in Driffill, Raybaudi, and Sola (2003), the reasons for these results are quite different.

An increase in $\sigma$, ceteris paribus, increases the option price, reflecting the fact that the greater is the risk, the greater is the option value. Nevertheless, this has the effect of increasing the critical value of the profit threshold and reducing the critical value of 
the project evaluated at the critical profits (because it reduces the convenience yields in both states). The overall effect on the value of the option would depend on all of these forces. In particular, we find that only the rise in $\sigma_{2}$ generates an increase in the option value in regime 2 evaluated at the threshold. On the contrary, the rise in $\sigma_{1}$ leads to a fall in the option values in both regimes. Likewise, an increase in $\sigma_{2}$ does not raise the value of the option to invest in regime 1, evaluated at the threshold.

To understand the weight in the final result of the different channels, notice that even though an increase in $\sigma_{i}$ has the effect of reducing the convenience yields - that is, the $k$-values (since the discount rate is a function of $\sigma_{i}$ ) - the final effect of an increase in $\sigma_{2}$ on $V_{2}\left(\pi_{2}^{*}\right)$ and $F_{2}\left(\pi_{2}^{*}\right)$ is positive. This is because the increase in the critical level of profits, $\pi_{2}^{*}$ (induced by the direct effect of an increase in the option value as a result of an increase in $\sigma_{2}$ ), outweighs the fall in $k_{2}$ and thus the value of the project in state $2, V_{2}\left(\pi_{2}^{*}\right)$, increases. From this, it follows that $F_{2}\left(\pi_{2}^{*}\right)$ also increases.

On the other hand, increasing the variance, $\sigma_{1}$, reduces the critical value of the project at entry in both states.-(this is due to the fact that the fall in $k_{i}$ outweighs the increase in $\pi_{i}^{*}$ ) Thus, the value of the option to invest (at the critical profit level) is decreasing in the innovation variance in all cases.

We now allow for changes in the expected durations of booms and recessions, i.e., changes in $h_{i}$. They are shown in the seventh and eighth rows of Table 6 . We observe that increasing the probability of switching to a recession regime $\left(h_{1}\right)$ affects negatively all the key variables, except the critical value of profits, and therefore it postpones the expected timing of the investment. We obtain opposite effects by increasing the probability of switching to a boom regime. Notice that these are the aggregate effects of accounting for different means and variances and discount factors associated with each regime. In particular, regime 1 has high drift and low variance while regime 2 has lower mean and higher variance. The final result depends on all of these forces. 
When we increase the probability of switching to a recession, the critical values of the projects at the thresholds decrease as a result of the lower expected profits in the recession state. Notice that when we observe the critical level of profits we see that the inaction region increases in both states as a result of the higher variance of the recession state. While the probability of switching to a boom increases, the value of the project evaluated at the critical level increases as a result of the higher drift in the boom.

Finally, we examine the effects of increases in $\Gamma^{\prime}$ s on the key variables. From the seventh and eighth rows of Table 6 we observe that all the key variables except the critical value of the profit have negative elasticities. Increasing $\Gamma^{\prime}$ s corresponds to changing the probability of regime-switching. 
Table 5: Sensitivity Analysis

\begin{tabular}{c|cccccc}
\hline \hline & \multicolumn{5}{c|}{ Small $\Gamma_{1}, \Gamma_{2}$} & \multicolumn{3}{c}{ Large $\Gamma_{1}, \Gamma_{2}$} \\
\cline { 2 - 7 } & $\Gamma_{1}=\Gamma_{2}$ & $\Gamma_{1}>\Gamma_{2}$ & $\Gamma_{1}<\Gamma_{2}$ & $\Gamma_{1}=\Gamma_{2}$ & $\Gamma_{1}>\Gamma_{2}$ & $\Gamma_{1}<\Gamma_{2}$ \\
\hline \multicolumn{7}{c}{ Regime-Switching Risk Premium } \\
\hline$\left.h_{1} \Gamma_{1}\right)$ & 0.020 & 0.038 & 0.020 & 0.116 & 0.224 & 0.109 \\
$h_{2} \Gamma_{2}$ & 0.020 & 0.020 & 0.035 & 0.116 & 0.116 & 0.132 \\
\hline$\pi_{1}^{*}$ & 2.21 & 2.40 & 2.14 & 2.68 & 2.96 & 0.72 \\
$\pi_{2}^{*}$ & 5.42 & 5.43 & 5.33 & 5.02 & 5.06 & 0.55 \\
\hline $\mathrm{k}$ Value & 47.23 & 39.55 & 53.58 & 37.89 & 30.76 & 46.71 \\
\hline$k_{1}$ & 34.82 & 29.98 & 41.00 & 34.39 & 28.29 & 43.14 \\
$k_{2}$ & 104.47 & 95.08 & 114.57 & 101.55 & 91.06 & 33.46 \\
\hline Project Value & 188.58 & 162.77 & 218.33 & 172.58 & 143.07 & 23.63 \\
\hline$V\left(\pi_{1}^{*}\right)$ & &
\end{tabular}

This table shows the value of the variable named in the left-hand entry of each row calculated under different combinations of $\Gamma_{1}, \Gamma_{2}$ values given at the head of each column. The combinations are as follows. The first three combinations are made under small values of $\Gamma_{1}, \Gamma_{2}$. The first case simply sets $\Gamma_{1}=\Gamma_{2}$. The second doubles the values of $\Gamma_{1}$, keeping the value of $\Gamma_{2}$ intact while the third doubles the values of $\Gamma_{2}$, keeping the value of $\Gamma_{1}$ intact. The combinations under large values are made by multiplying the benchmark values of $\Gamma_{1}, \Gamma_{2}$ by either 20 or 30: The first takes 20 times the benchmark values of $\Gamma_{1}, \Gamma_{2}$; the second combines 30 times the benchmark value of $\Gamma_{1}$ with 20 times the benchmark value of $\Gamma_{2}$ while the last combines 20 times the benchmark value of $\Gamma_{1}$ with 30 times the benchmark value of $\Gamma_{2}$. The quantities whose sensitivities are shown are as follows: $\pi_{1}^{*}=$ critical value of the profit level that triggers investment in regime 1 (expansion); $\pi_{2}^{*}=$ critical value of the profit level that triggers investment in regime 2 (recession); $k_{1}^{*}=$ convenience yield in regime $1 ; k_{2}^{*}=$ convenience yield in regime $2 ; V\left(\pi_{1}^{*}\right)=$ the critical value of the project in regime 1 ; and $V\left(\pi_{2}^{*}\right)=$ the critical value of the project in regime 2 .

This numerical analysis is based on the baseline values for all the parameters except $I=50$ are given in Table 1. 
Table 6: Elasticities of Variables with Respect to the Key Parameters

(Two-State Regime-Switching Model with Regime Switching Risk)

\begin{tabular}{cc|cccccccccc}
\hline \hline & $\pi_{1}^{*}$ & $\pi_{2}^{*}$ & $k_{1}$ & $k_{2}$ & $h_{1} \Gamma_{1}$ & $h_{2} \Gamma_{2}$ & $V\left(\pi_{1}^{*}\right)$ & $V\left(\pi_{2}^{*}\right)$ & $F\left(\pi_{1}^{*}\right)$ & $F\left(\pi_{2}^{*}\right)$ \\
\hline$\mu_{1}$ & -1.030 & -0.077 & 1.175 & 0.759 & 1.669 & 1.115 & 0.133 & 0.682 & 0.335 & 1.148 \\
& $\mu_{2}$ & -0.094 & -0.056 & 0.126 & 0.182 & 0.286 & 0.192 & 0.031 & 0.125 & 0.080 & 0.211 \\
& $\rho_{1} \lambda_{1}$ & 0.202 & 0.015 & -0.225 & -0.145 & -0.326 & -0.219 & -0.023 & -0.130 & -0.058 & -0.219 \\
& $\rho_{2} \lambda_{2}$ & 0.226 & 0.360 & -0.346 & -0.500 & -0.064 & -0.043 & -0.121 & -0.142 & -0.305 & -0.239 \\
& $\sigma_{1}$ & 0.211 & 0.016 & -0.225 & -0.145 & -0.353 & -0.237 & -0.014 & -0.130 & -0.035 & -0.218 \\
& $\sigma_{2}$ & 0.310 & 0.712 & -0.346 & -0.500 & 0.750 & 0.503 & -0.038 & 0.209 & -0.095 & 0.351 \\
$\Gamma_{1}$ & 0.013 & 0.000 & -0.021 & -0.014 & -0.016 & -0.011 & -0.009 & -0.014 & -0.022 & -0.023 \\
$\Gamma_{2}$ & 0.007 & 0.002 & -0.015 & -0.022 & -0.034 & -0.023 & -0.009 & -0.020 & -0.022 & -0.033 \\
$h_{1}$ & 0.355 & 0.000 & -0.592 & -0.383 & -0.437 & -0.294 & -0.240 & -0.383 & -0.606 & -0.644 \\
$h_{2}$ & -0.115 & -0.040 & 0.262 & 0.378 & 0.582 & 0.390 & 0.146 & 0.338 & 0.371 & 0.569 \\
\hline \hline
\end{tabular}

This table shows the percentage change in the variable named at the head of each column caused by a unit percentage change in the variable named in the left-hand entry of each row. The quantities whose responses are shown are as follows: $\pi_{1}^{*}=$ critical value of the profit level that triggers investment in regime 1 (expansion); $\pi_{2}^{*}=$ critical value of the profit level that triggers investment in regime 2 (recession); $k_{1}^{*}=$ convenience yield in regime $1 ; k_{2}^{*}=$ convenience yield in regime $2 ; h_{1} \Gamma_{1}=$ regime-switching risk premium in regime $1 ; h_{2} \Gamma_{2}=$ regime-switching risk premium in regime $2 ; V\left(\pi_{1}^{*}\right)=$ the critical value of the project in regime $1 ; V\left(\pi_{2}^{*}\right)=$ the critical value of the project in regime 2 ; $F\left(\pi_{1}^{*}\right)=$ the value of the option to invest in regime 1 ; and $F\left(\pi_{2}^{*}\right)=$ the value of the option to invest in regime 2 .

This numerical analysis is based on the baseline values for all the parameters except $I=50$ are given in Table 1. 
Figure 1: Firm Values and Option Values in Two Regimes

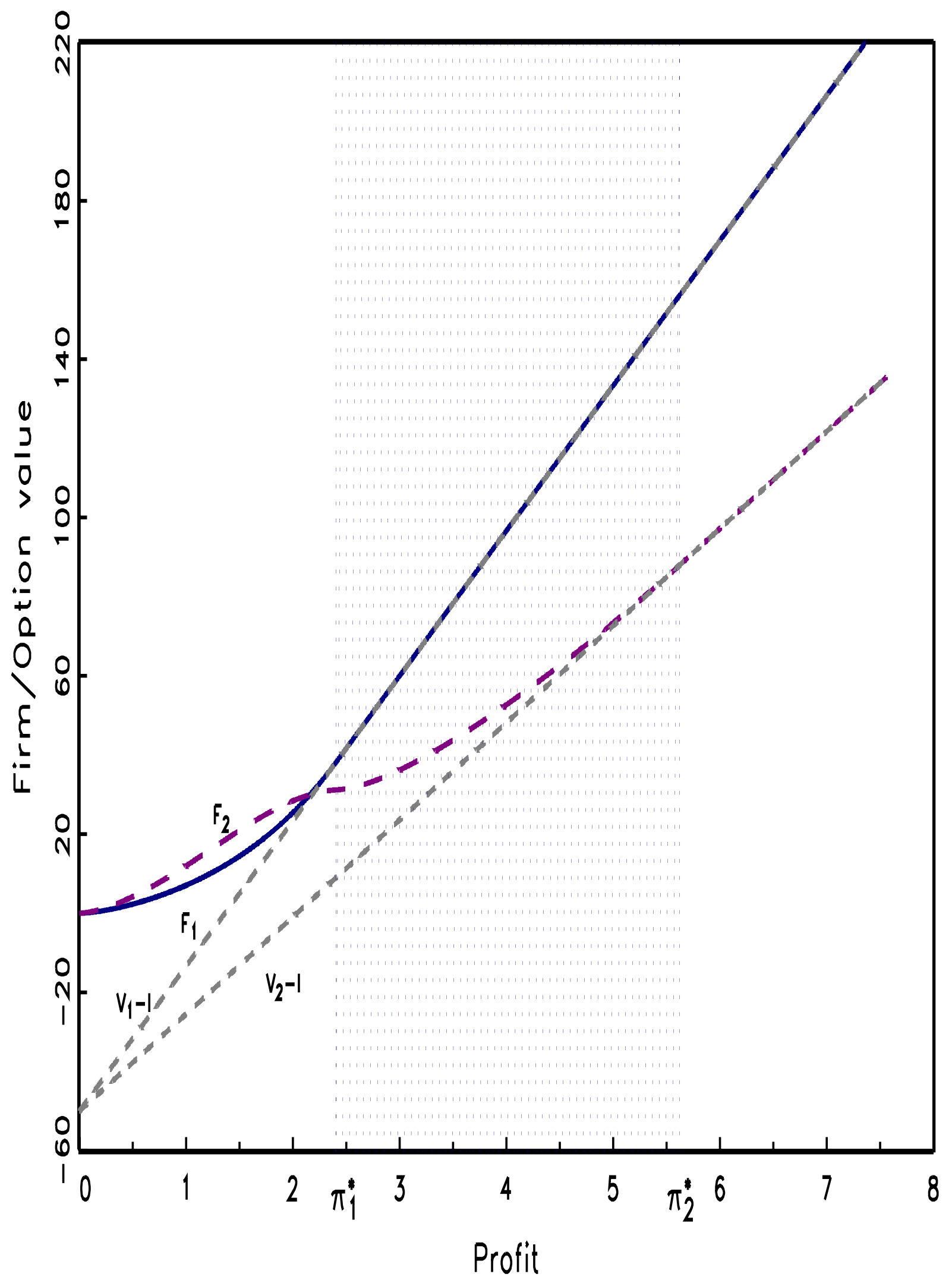




\section{Conclusion}

This paper has analysed investment decisions under uncertainty when the dynamics of both the decision variable - growth rate and volatility parameter - and the stochastic discount factor - price of factor risk and price of regime switching risk - shift between states at random times. We derive closed form investment policies for the manager which maximize the value of the project. As in Guo, Miao, and Morellec (2005) the value-maximizing investment policy is such that in each regime the firm's investment policy is optimal, conditional on the optimal investment policy in the other regimes. Because of the possibility of a regime shift, the option to invest in each regime reflects the possibility for the firm to invest in the other regime. The comparative statics revealed significant valuation effects, and altered the timing of exercise of the options. We find that incorporating regime-dependent diffusion risk precipitates the expected time of the investment in booms and postpones the expected time of investments in recessions. This nature of our model reinforces the finding of Guo, Miao, and Morellec (2005) that "investment is lumpy following a shift from the regime with the highest investment curve to the regime with the lowest one." Allowing for the Markov-switching price of the risk on the other hand has the effect of postponing the expected time of the investment in both regimes. This is due to the fact that adding this extra component to the discount factor modifies the critical thresholds but does not modify the driving process. This strikes a clear distinction from the recent papers on regime switching real options.

Some possible extensions of the framework would be: Firstly, the model can be generalized to more than two states, secondly, the model can be extended to a general equilibrium model of investment under uncertainty, thirdly, the model can be used to estimate as well as to calibrate the observed factor and regime-switching risk premia. These might be directions for future research. 


\section{Appendix: Derivations and Proofs for Propo- sitions}

\section{Appendix A. Derivation of Equations (8) and (9)}

\section{Project valuation.}

Following Cochrane (2005) the no-arbitrage risky project valuation equation can be written as

$$
0=M\left(s_{t}\right) \pi_{t} d t+E\left[d\left(M\left(s_{t}\right) V\left(\pi_{t}, s_{t}\right)\right)\right] .
$$

We apply Ito's lemma to break up the last term $d\left(M\left(s_{t}\right) V\left(\pi_{t}, s_{t}\right)\right)$ :

$$
d\left(M\left(s_{t}\right) V\left(\pi_{t}, s_{t}\right)\right)=V\left(\pi_{t}, s_{t}\right) d M\left(s_{t}\right)+M\left(s_{t}\right) d V\left(\pi_{t}, s_{t}\right)+d M\left(s_{t}\right) d V\left(\pi_{t}, s_{t}\right) .
$$

For this regime-switching diffusion setting, following the work of Dai and Singleton (2003) the process $\left\{M\left(s_{t}\right)\right\}$ can be written as follows:

$$
d M\left(s_{t}\right)=-r^{f} M\left(s_{t}\right) d t-\lambda_{s_{t}} M\left(s_{t}\right) d Z_{t}-M\left(s_{t}\right) \Gamma_{s_{t}} d N_{t}
$$

where $r^{f}$ is the risk-free rate of return, $\lambda_{s_{t}}$ is the (regime-dependent) market price of continuous risk (diffusion risk), $\Gamma_{s_{t}}$ is the market price of a shift from regime $s_{t}=j$ to regime $i(i \neq j ; i, j=1,2)$, and $d Z_{t}$ is the increment of a standard Wiener process, which is correlated with $d W_{t}$ with correlation coefficient $\rho_{s_{t}}$ (the correlation between returns of the market portfolio and the cash flows of the project).

By substituting Eq. (A.3) for $d M$ in Eq. (A.1) we obtain the following expression:

$$
r^{f} V\left(\pi_{t}, s_{t}\right) d t=\pi_{t} d t+E\left[d V\left(\pi_{t}, s_{t}\right)\right]+E\left[\frac{d M\left(s_{t}\right) d V\left(\pi_{t}, s_{t}\right)}{M\left(s_{t}\right)}\right] .
$$

To obtain the final result presented in the text we need to derive an expression for $E\left[d V\left(\pi_{t}, s_{t}\right)\right]$ and $E\left[\frac{d M\left(s_{t}\right) d V\left(\pi_{t}, s_{t}\right)}{M\left(s_{t}\right)}\right]$. 
i) Derivation of $E d V\left(\pi_{t}, s_{t}\right)$.

Let $V_{i}=V\left(\pi_{t}, e_{i}\right)$ for $i=1,2$ and $V\left(\pi_{t}, s_{t}\right)=\left\langle\vec{V}, s_{t}\right\rangle$ where $\vec{V}=\left(V_{1}, V_{2}\right)$ is a $2 \times 1$ row vector consisting of elements $V_{1}, V_{2}$. Here $\langle.,$.$\rangle is the inner product operator used$ in the following way: If $x, y$ are (column) vectors in $\mathbb{R}^{N}$ we write $\langle x, y\rangle=x^{\prime} y$ for their scalar (inner) product. When $x$ is a matrix and $y$ is a (column) vector, we write we write $\langle x, y\rangle=\operatorname{diag}(x y)$ denotes the diagonal matrix with vector $x y$ on its diagonal.

Then using Ito's Lemma to cases with regime shifts along the lines of Elliot, Aggoun, and Moore (1995) we can express the change in the project value as to $d V=d V\left(\pi_{t}, s_{t}\right)$ as in Elliott, Miao, and Yu (2007):

$$
\begin{aligned}
d V= & \langle d \vec{V}, s\rangle+\langle\vec{V}, d s\rangle \\
= & V_{\pi}\left(\pi_{t}, s_{t}\right) d \pi+\frac{1}{2} V_{\pi \pi}\left(\pi_{t}, s_{t}\right)(d \pi)^{2}+\left\langle\vec{V}, H s_{t} d t\right\rangle+\langle\vec{V}, d N\rangle, \\
= & \underbrace{\left(\mu_{s_{t}} V_{\pi}\left(\pi_{t}, s_{t}\right) \pi+\frac{1}{2} \sigma_{s_{t}}^{2} V_{\pi \pi}\left(\pi_{t}, s_{t}\right) \pi^{2}\right) d t+\sigma_{s_{t}} V_{\pi}\left(\pi_{t}, s_{t}\right) \pi d W_{t}}_{\text {due to diffusion }}+ \\
& +\underbrace{\left\langle\vec{V}, H s_{t} d t\right\rangle+\langle\vec{V}, d N\rangle}_{\text {due to discrete shifts }}
\end{aligned}
$$

where the subscripts $\pi$ and $\pi \pi$ denote, respectively, the first and second partial derivatives of variable $V$ with respect to $\pi$.

Notice that we can express $\left\langle\vec{V}, H s_{t} d t\right\rangle=\left(h_{1}\left[V_{2}-V_{1}\right] d t, h_{2}\left[V_{1}-V_{2}\right] d t\right)^{\prime}$ and, using the fact that $d N=\left(d N_{1}, d N_{2}\right)$ and that $d N_{1}=-d N_{2}$, we can write $\langle\vec{V}, d N\rangle=$ $\left[V_{1}-V_{2}\right] d N_{1}$.

This gives the following two equations when $s_{t}=e_{1}$ or $s_{t}=e_{2}$,

$$
\begin{aligned}
& d V_{1}=\left(\mu_{1} V_{1 \pi} \pi+\frac{1}{2} \sigma_{1}^{2} V_{1 \pi \pi} \pi^{2}+h_{1}\left[V_{2}-V_{1}\right]\right) d t+\sigma_{1_{t}} V_{1 \pi} \pi d W+\left[V_{1}-V_{2}\right] d N_{1} \\
& d V_{2}=\left(\mu_{2} V_{2 \pi} \pi+\frac{1}{2} \sigma_{2}^{2} V_{2 \pi \pi} \pi^{2}+h_{2}\left[V_{1}-V_{2}\right]\right) d t+\sigma_{2_{t}} V_{2 \pi} \pi d W+\left[V_{2}-V_{1}\right] d N_{2}
\end{aligned}
$$

By taking expectations we obtain

$$
\begin{aligned}
& E\left(d V_{1}\right)=\left(\mu_{1} V_{1 \pi} \pi+\frac{1}{2} \sigma_{1}^{2} V_{1 \pi \pi} \pi^{2}+h_{1}\left[V_{2}-V_{1}\right]\right) d t, \\
& E\left(d V_{2}\right)=\left(\mu_{2} V_{2 \pi} \pi+\frac{1}{2} \sigma_{2}^{2} V_{2 \pi \pi} \pi^{2}+h_{2}\left[V_{1}-V_{2}\right]\right) d t .
\end{aligned}
$$


ii) Derivation of $E\left(\frac{d M\left(s_{t}\right) d V\left(\pi_{t}, s_{t}\right)}{M\left(s_{t}\right)}\right)$.

To arrive to the final solution of this expression we start by deriving, for each regime an expression of the product of the product of $d M\left(s_{t}\right) d V\left(\pi_{t}, s_{t}\right)$.

$$
\begin{aligned}
& d M\left(e_{1}\right) d V_{1}=-\lambda_{1} \rho \sigma_{1_{t}} M\left(e_{1}\right) V_{1 \pi} \pi d t-M\left(e_{1}\right) \Gamma_{1}\left[V_{1}-V_{2}\right] d N_{1}^{2} \\
& d M\left(e_{2}\right) d V_{2}=-\lambda_{2} \rho \sigma_{2_{t}} M\left(e_{2}\right) V_{2 \pi} \pi d t-M\left(e_{2}\right) \Gamma_{2}\left[V_{2}-V_{1}\right] d N_{2}^{2}
\end{aligned}
$$

Notice that $E\left(d W_{t} d Z_{t}\right)=\rho d t$ that an expression $(d N)^{2}$ can be obtained using the results presented in Lemma 1.3 in Appendix B of Elliot, Aggoun, and Moore (1995):

$$
(d N)^{2}=\operatorname{diag}\left(H s_{t}\right) d t-\operatorname{diag}\left(s_{t}\right) H^{\prime} d t-H \operatorname{diag}\left(s_{t}\right) d t
$$

where $\operatorname{diag}(x)$ denotes the diagonal matrix with vector $x$ on its diagonal. This expression simplifies to $(d N)^{2}=\operatorname{diag}\left(\left(h_{1} d t, h_{2} d t\right)^{\prime}\right)$.

Substituting this result in Eq. (A.6) yields:

$$
\begin{aligned}
& E\left(\frac{d M\left(e_{1}\right) d V_{1}}{M\left(e_{1}\right)}\right)=-\lambda_{1} \rho \sigma_{1} V_{1 \pi} \pi d t-\Gamma_{1} h_{1}\left[V_{1}-V_{2}\right] d t, \\
& E\left(\frac{d M\left(e_{2}\right) d V_{2}}{M\left(e_{2}\right)}\right)=-\lambda_{1} \rho \sigma_{1} V_{1 \pi} \pi d t-\Gamma_{2} h_{2}\left[V_{2}-V_{1}\right] d t .
\end{aligned}
$$

By substituting this expression in

$$
r^{f} V\left(\pi_{t}, s_{t}\right) d t=\pi_{t} d t+E d V\left(\pi_{t}, s_{t}\right)+E \frac{d M\left(s_{t}\right) d V\left(\pi_{t}, s_{t}\right)}{M\left(s_{t}\right)} .
$$

gives us

$$
\begin{aligned}
& r^{f} V_{1}=\pi+\left(\mu_{1}-\lambda_{1} \rho \sigma_{1}\right) V_{1 \pi} \pi+\frac{1}{2} \sigma_{1}^{2} V_{1 \pi \pi} \pi^{2}+h_{1}\left(1+\Gamma_{1}\right)\left[V_{2}-V_{1}\right] \\
& r^{f} V_{2}=\pi+\left(\mu_{2}-\lambda_{2} \rho \sigma_{2}\right) V_{2 \pi} \pi+\frac{1}{2} \sigma_{2}^{2} V_{2 \pi \pi} \pi^{2}+h_{2}\left(1+\Gamma_{2}\right)\left[V_{1}-V_{2}\right]
\end{aligned}
$$

which is Eq. (14) in the main text. 


\section{Appendix B. Proof of Proposition 1}

We rewrite the second-order differential equations to be solved conditional on $s_{t}=e$ :

$$
\pi+\left[\mu_{s_{t}}-\lambda_{s_{t}} \rho_{s_{t}} \sigma_{s_{t}}\right] V_{\pi} \pi+\frac{1}{2} \sigma_{s_{t}}^{2} V_{\pi \pi} \pi^{2}+\left\langle V, H s_{t}\right\rangle-r^{f} V-\Gamma_{s_{t}}\left\langle V, H s_{t}\right\rangle=0
$$

Writing explicitly the above equation yields:

$$
\begin{aligned}
& \pi+\left[\mu_{1}-\lambda_{1} \rho_{1} \sigma_{1}\right] V_{1 \pi} \pi+\frac{1}{2} \sigma_{1}^{2} V_{1 \pi \pi} \pi^{2}+h_{1}\left(1-\Gamma_{1}\right)\left(V_{2}-V_{1}\right)-r^{f} V_{1}=0 \\
& \pi+\left[\mu_{2}-\lambda_{2} \rho_{2} \sigma_{2}\right] V_{2 \pi} \pi+\frac{1}{2} \sigma_{2}^{2} V_{2 \pi \pi} \pi^{2}+h_{2}\left(1-\Gamma_{2}\right)\left(V_{1}-V_{2}\right)-r^{f} V_{2}=0
\end{aligned}
$$

where $V_{1}=V\left(\pi_{t}, e_{1}\right)$ and $V_{2}=V\left(\pi_{t}, e_{2}\right)$. The general solutions to these equations consist of two parts: $(i)$ the solution to the characteristic function and $(i i)$ the particular integral. The solutions to the characteristic function are identified with bubbles in the asset price and have to be equal to zero if the asset price is to equal the present value of future returns. The particular integrals, which equal the present value of future returns, are

$$
V_{s t}=k_{s t} \pi
$$

where $k_{s t}$ is either $k_{1}$ when $e_{1}$ or $k_{2}$ when $e_{2}$, which are constants to be determined. By defining $r_{i}^{e}=r^{f}+\lambda_{i} \rho_{i} \sigma_{i}$ and substituting (B.2) together with its first and second derivatives with respect to $\pi$ into (B.1), we obtain a pair of equations

$$
\begin{aligned}
& r_{1}^{e} k_{1}=1+k_{1} \mu_{1}+\hat{h}_{1}\left(k_{2}-k_{1}\right), \\
& r_{2}^{e} k_{2}=1+k_{2} \mu_{2}+\hat{h}_{2}\left(k_{1}-k_{2}\right),
\end{aligned}
$$

which can be solved for $k_{1}$ and $k_{2}$ :

$$
\begin{aligned}
& k_{1}=\left[r_{2}^{e}+\hat{h}_{1}+\hat{h}_{2}-\mu_{2}\right] / \Omega, \\
& k_{2}=\left[r_{1}^{e}+\hat{h}_{1}+\hat{h}_{2}-\mu_{1}\right] / \Omega,
\end{aligned}
$$

where $\Omega=\left(r_{1}^{e}+\hat{h}_{1}-\mu_{1}\right)\left(r_{2}^{e}+\hat{h}_{2}-\mu_{2}\right)-\hat{h}_{1} \hat{h}_{2}$. These equations may be written in a compact form as in Eq. (15b) in the main text. 


\section{Appendix C. Proof of Proposition 2}

As noted in the text, there are three investment regions of interest. These regions are determined by the critical trigger levels corresponding to the two regimes, $\pi_{1}^{*}$ and $\pi_{2}^{*}$.

\section{C.1 Investment Problem in (Common Inaction) Region 1}

Consider Region 1 where $\pi \leq \pi_{1}^{*}$. In this region, no investment is made regardless of the level of profits. The value of the option to invest, $F\left(\pi_{t}, s_{t}\right)$, can be obtained from the basic asset pricing expression

$$
E\left[d\left(M\left(s_{t}\right) F\left(\pi_{t}, s_{t}\right)\right)\right]=0
$$

which is isomorphic with equation (A.1) with the profit stream set to zero, and $F($. replaces $V($.$) . Consequently the solution for (C.1) mirrors that for (A.1). The result$ is identical to equation (A.9), except that the first term on the right-hand side of (A.9) $(\pi)$ does not appear, and we have:

$$
\begin{aligned}
& \mu_{1} F_{1 \pi} \pi+\frac{1}{2} \sigma_{1}^{2} F_{1 \pi \pi} \pi^{2}+h_{1}\left(1-\Gamma_{1}\right)\left(F_{2}-F_{1}\right)-r^{f} F_{1}-\rho_{1} \lambda_{1} \sigma_{1} \pi F_{1}=0 \\
& \mu_{2} F_{2 \pi} \pi+\frac{1}{2} \sigma_{2}^{2} F_{2 \pi \pi} \pi^{2}+h_{2}\left(1-\Gamma_{2}\right)\left(F_{1}-F_{2}\right)-r^{f} F_{2}-\rho_{2} \lambda_{2} \sigma_{2} \pi F_{2}=0,
\end{aligned}
$$

where $F_{\pi}$ and $F_{\pi \pi}$ denote, respectively, the first and second partial derivatives of $F$ with respect to $\pi$ while the subscripts 1 and 2 denote the partial derivatives taken in regimes 1 and 2, respectively.

\section{C.2 Solution for Investment Problem in Region 1}

Assuming that $F_{1}$ and $F_{2}$ are linearly independent, the trial solutions of Eq. (C.2) take the following two equations when $s_{t}=e_{1}$ or $s_{t}=e_{2}$,

$$
\begin{aligned}
& F_{1}=A \pi_{1}^{\gamma}, \\
& F_{2}=B \pi_{2}^{\gamma},
\end{aligned}
$$


where $A$ and $B$ are constants to be determined. Substituting these solutions into Eq. (C.2) and matching coefficients yields the following expressions:

$$
\begin{aligned}
& {\left[r_{1}^{e}+\hat{h}_{1}-\mu_{1} \gamma-\frac{1}{2} \sigma_{1}^{2} \gamma(\gamma-1)\right] A=h_{1} B,} \\
& {\left[r_{2}^{e}+\hat{h}_{2}-\mu_{2} \gamma-\frac{1}{2} \sigma_{2}^{2} \gamma(\gamma-1)\right] B=h_{2} A,}
\end{aligned}
$$

where $\hat{h}_{i}=h_{i}\left(1-\Gamma_{i}\right)$. We can eliminate $A$ and $B$ from these two equations and obtain a fourth order polynomial in $\gamma$, thus:

$$
\left[r_{1}^{e}+\hat{h}_{1}-\mu_{1} \gamma-\frac{1}{2} \sigma_{1}^{2} \gamma(\gamma-1)\right]\left[r_{2}^{e}+\hat{h}_{2}-\mu_{2} \gamma-\frac{1}{2} \sigma_{2}^{2} \gamma(\gamma-1)\right]=h_{1} h_{2}
$$

This equation has four distinct roots, with $\gamma_{3}<\gamma_{4}<0$ and $1<\gamma_{1}<\gamma_{2}$. The general solution to (C.2) is:

$$
\begin{aligned}
& F_{1}=\sum_{i=1}^{4} A_{i} \pi^{\gamma_{i}}, \\
& F_{2}=\sum_{i=1}^{4} B_{i} \pi^{\gamma_{i}} .
\end{aligned}
$$

However, since $F_{i}(0)=0$ can only be satisfied if $A_{3}=A_{4}=B_{3}=B_{4}=0$, the solution reduces to:

$$
\begin{aligned}
& F_{1}=\sum_{i=1}^{2} A_{i} \pi^{\gamma_{i}}, \\
& F_{2}=\sum_{i=1}^{2} B_{i} \pi^{\gamma_{i}} .
\end{aligned}
$$

\section{C.3 Investment Problem in Region 2}

In Region 2, the firm only invests if $\pi>\pi_{1}^{*}$. The basic asset pricing used above is modified slightly to account for the investment taking place if the economy is in regime 1:

$$
\begin{aligned}
& F_{1} \leq V_{1}-I, \\
& \mu_{1} \pi_{1} F_{2 \pi}+\frac{1}{2} \sigma_{1}^{2} \pi_{2}^{2} F_{2 \pi \pi}+\tilde{h}_{2}\left(F_{1}-F_{2}\right)-r^{f} F_{2}-\rho_{2} \lambda_{2} \sigma_{2} \pi_{2} F_{2 \pi}=0 .
\end{aligned}
$$

Substituting and simplifying yields

$$
\mu_{2} \pi_{2} F_{2 \pi}+\frac{1}{2} \sigma_{2}^{2} \pi_{2}^{2} F_{2 \pi \pi}+\hat{h}_{2}\left(V_{1}-I-F_{2}\right)-r^{f} F_{2}-\rho_{2} \lambda_{2} \sigma_{2} \pi_{2} F_{2 \pi}=0
$$


Given that $V_{1}(\pi)=k_{1} \pi$, we rewrite Eq. (C.6) as

$$
\mu_{2} \pi_{2} F_{2 \pi}+\frac{1}{2} \sigma_{2}^{2} \pi_{2}^{2} F_{2 \pi \pi}-\hat{h}_{2} F_{2}-r^{f} F_{2}-\rho_{2} \lambda_{2} \sigma_{2} \pi_{2} F_{2 \pi}=\hat{h}_{2}\left(I-k_{1} \pi\right) .
$$

The trial solution to the corresponding homogenous part of Eq. (C.7) takes the form

$$
F_{2}^{c}(\pi)=C \pi^{\zeta}
$$

where the $\zeta^{\prime} s$ satisfy the following quadratic equation

$$
\mu_{2} \zeta+\frac{\sigma_{2}^{2}}{2} \zeta(\zeta-1)-\left(\hat{h}_{2}+r_{2}^{e}\right)=0 .
$$

With the real and unequal roots of the quadratic equation of (C.9) the general solution of the homogenous part of (C.7) is

$$
F_{2}^{c}(\pi)=C_{1} \pi^{\zeta_{1}}+C_{2} \pi^{\zeta_{2}}
$$

where $\zeta_{1}<0$ and $\zeta_{2}>1$.

Using the method of undetermined coefficients, one can show that the forcing term $\hat{h}_{2}\left(I-k_{1} \pi\right)$ of the non-homogeneous equation (C.7) takes the following particular solution

$$
F_{2}^{n c}(\pi)=C_{3} \pi+C_{4}
$$

By summing up the two solutions $F^{c}$ and $F^{n c}$, we obtain the general solution to (C.7) as

$$
F_{2}=C_{1} \pi^{\zeta_{1}}+C_{2} \pi^{\zeta_{2}}+C_{3} \pi+C_{4}
$$

To determine $C_{3}$ and $C_{4}$ in the particular solution, we differentiate the candidate solution Eq. (C.10) and plug it into Eq. (C.7) to obtain

$$
\mu_{2} C_{3} \pi-\left(\hat{h}_{2}+r_{2}^{e}\right)\left(C_{3} \pi+C_{4}\right)=\hat{h}_{2} I-\hat{h}_{2} k_{1} \pi .
$$


Applying the constant coefficients technique gives us

$$
\begin{aligned}
\mu_{2} C_{3}-\left(\hat{h}_{2}+r_{2}^{e}\right) C_{3} \pi & =-\hat{h}_{2} k_{1}, \\
-\left(\hat{h}_{2}+r_{2}^{e}\right) C_{4} & =\hat{h}_{2} I .
\end{aligned}
$$

The solutions of these equations are

$$
\begin{aligned}
C_{3} & =\frac{\hat{h}_{2} k_{1}}{\left(\hat{h}_{2}+r_{2}^{e}\right)-\mu_{2}}, \\
C_{4} & =-\frac{\hat{h}_{2} I}{\left(\hat{h}_{2}+r_{2}^{e}\right)} .
\end{aligned}
$$

Therefore we finally obtain the solution for $F_{2}$ :

$$
F_{2}(\pi)=C_{1} \pi^{\zeta_{1}}+C_{2} \pi^{\zeta_{2}}-\frac{\hat{h}_{2} I}{\left(\hat{h}_{2}+r_{2}^{e}\right)}+\frac{\hat{h}_{2} k_{1}}{\left(\hat{h}_{2}+r_{2}^{e}\right)-\mu_{2}} \pi
$$

\section{C.4 Investment Problem in Region 3}

Finally, in Region 3, investment is made. The value functions are given by

$$
\begin{aligned}
& F_{1} \leq V_{1}-I \\
& F_{2} \leq V_{2}-I .
\end{aligned}
$$

\section{C.5 Solution of the Constants and Critical Triggers}

In order to solve the above equations for the constants $A_{i}, B_{i}, C_{i}(i=1,2)$ and critical triggers $\pi_{i}^{*}(i=1,2)$ we utilize the fact that the value of investment opportunity $F_{i}(i,=1,2)$ must also satisfy the following boundary conditions:

(i) the value-matching conditions

$$
F_{i}\left(\pi_{i}^{*}\right)=V_{i}\left(\pi_{i}^{*}\right)-I, \quad i=1,2
$$


(ii) the smooth-pasting conditions

$$
F_{i}^{\prime}\left(\pi_{i}^{*}\right)=V_{i}^{\prime}\left(\pi^{*}\right), \quad i=1,2
$$

(iii) the continuity value-matching condition

$$
\lim _{\pi \downarrow \pi_{1}^{*}} F_{2}(\pi)=\lim _{\pi \uparrow \pi_{1}^{*}} F_{2}(\pi),
$$

(iv) and the continuity smooth-pasting condition

$$
\lim _{\pi \downarrow \pi_{1}^{*}} F_{2}^{\prime}(\pi)=\lim _{\pi \uparrow \pi_{1}^{*}} F_{2}^{\prime}(\pi) .
$$

More precisely, we have from the value-matching conditions

$$
\begin{aligned}
& A_{1} \pi_{1}^{* \gamma_{1}}+A_{2} \pi_{1}^{* \gamma_{2}}=k_{1} \pi_{1}^{*}-I, \\
& -\frac{\hat{h}_{2} I}{\left(\hat{h}_{2}+r_{2}^{e}\right)}+\frac{\tilde{h}_{2} k_{1}}{\left(\hat{h}_{2}+r_{2}^{e}\right)-\mu_{2}} \pi_{2}^{*}+C_{1} \pi_{2}^{* \zeta_{1}}+C_{2} \pi_{2}^{* \zeta_{2}}=k_{2} \pi_{2}^{*}-I,
\end{aligned}
$$

from the smooth-pasting conditions

$$
\begin{aligned}
& \gamma_{1} A_{1} \pi_{1}^{*\left(\gamma_{1}-1\right)}+\gamma_{2} A_{2} \pi_{1}^{*\left(\gamma_{2}-1\right)}=k_{1}, \\
& \frac{\hat{h}_{2} k_{1}}{\left(\hat{h}_{2}+r_{2}^{e}\right)-\mu_{2}}+\zeta_{1} C_{1} \pi_{2}^{*\left(\zeta_{1}-1\right)}+\zeta_{2} C_{2} \pi_{2}^{*\left(\zeta_{2}-1\right)}=k_{1},
\end{aligned}
$$

and from continuity value-matching and smooth-pasting conditions

$$
\begin{gathered}
B_{1} \pi_{1}^{* \gamma_{1}}+B_{2} \pi_{1}^{* \gamma_{2}}=-\frac{\hat{h}_{2} I}{\left(\hat{h}_{2}+r_{2}^{e}\right)}+\frac{\hat{h}_{2} k_{1}}{\left(\hat{h}_{2}+r_{2}^{e}\right)-\mu_{2}} \pi_{1}^{*}+C_{1} \pi_{1}^{* \zeta_{1}}+C_{2} \pi_{1}^{* \zeta_{2}}, \\
\gamma_{1} B_{1} \pi_{1}^{*\left(\gamma_{1}-1\right)}+\gamma_{2} B_{2} \pi_{1}^{*\left(\gamma_{2}-1\right)}=\frac{\hat{h}_{2} k_{1}}{\left(\hat{h}_{2}+r_{2}^{e}\right)-\mu_{2}}+\zeta_{1} C_{1} \pi_{1}^{*\left(\zeta_{1}-1\right)}+\zeta_{2} C_{2} \pi_{1}^{*\left(\zeta_{2}-1\right)} .
\end{gathered}
$$


This is a system of 6 equations (C.13a)-C.13d) in 8 unknowns $\left(\pi_{1}^{*}, \pi_{2}^{*}, A_{1}, A_{2}, B_{1}, B_{2}\right.$, $\left.C_{1}, C_{2}\right)$. To eliminate $\left(B_{1}, B_{2}\right)$ we use Eq. (C.4) as follows:

$$
\begin{aligned}
& G_{0}\left(\gamma_{1}\right) A_{1}=h_{2}\left(1-\Gamma_{2}\right) B_{1}, \\
& G_{0}\left(\gamma_{2}\right) A_{2}=h_{1}\left(1-\Gamma_{1}\right) B_{2}, \\
& G_{1}\left(\gamma_{1}\right) B_{1}=h_{2}\left(1-\Gamma_{2}\right) A_{1}, \\
& G_{1}\left(\gamma_{2}\right) B_{2}=h_{2}\left(1-\Gamma_{2}\right) A_{2},
\end{aligned}
$$

where

$$
G_{i}\left(\gamma_{j}\right)=\left[r_{i}^{e}+h_{i}\left(1-\Gamma_{j}\right)-\mu_{i} \gamma_{j}-\frac{1}{2} \sigma_{i}^{2} \gamma_{j}\left(\gamma_{j}-1\right)\right], \quad i=1,2, \quad j=1,2
$$

Solving the above four equations (C.14a)-(C.14d) for $B_{1}$ and $B_{2}$ yields

$$
B_{1}=l_{1} A_{1}, ; \quad B_{2}=l_{2} A_{2},
$$

where $l_{1}=\frac{G_{0}\left(\gamma_{1}\right)}{h_{1}\left(1-\Gamma_{1}\right)}=\frac{h_{2}\left(1-\Gamma_{2}\right)}{G_{1}\left(\gamma_{1}\right)}$ and $l_{2}=\frac{G_{0}\left(\gamma_{2}\right)}{h_{1}\left(1-\Gamma_{1}\right)}=\frac{h_{2}\left(1-\Gamma_{2}\right)}{G_{1}\left(\gamma_{2}\right)}$.

From Eqs. (C.13a) and (C.13c) we obtain expressions for $A_{1}$ and $A_{2}$ :

$$
A_{1}=\frac{\left(\gamma_{2}-1\right) k_{1} \pi_{1}^{*}-\gamma_{2} I}{\left(\gamma_{2}-\gamma_{1}\right)\left(\pi_{1}^{*}\right)^{\gamma_{1}}} ; \quad A_{2}=\frac{\left(\gamma_{1}-1\right) k_{1} \pi_{1}^{*}-\gamma_{1} I}{\left(\gamma_{1}-\gamma_{2}\right)\left(\pi_{1}^{*}\right)^{\gamma_{2}}}
$$

Similarly, Eqs. (C.13b and (C.13d yield expressions for $C_{1}$ and $C_{2}$ :

$$
\begin{aligned}
C_{1}= & \frac{\zeta_{2} \frac{r_{2}^{e} I}{\left[\hat{h}_{2}+r_{2}^{e}\right]}+\left(1-\zeta_{2}\right)\left[\frac{\hat{h}_{2}}{\left(\hat{h}_{2}+r_{2}^{e}\right)-\mu_{2}} k_{1}-k_{2}-\frac{\zeta_{2}}{\left(1-\zeta_{2}\right)} I\right] \pi_{2}}{\left(\pi_{2}^{*}\right)^{\zeta_{1}}\left(\zeta_{2}-\zeta_{1}\right)}, \\
C_{2}= & \frac{\zeta_{1} \frac{r_{2}^{e} I}{\left[\hat{h}_{2}+r_{2}^{e}\right]}+\left(1-\zeta_{1}\right)\left[\frac{\hat{h}_{2}}{\left(\hat{h}_{2}+r_{2}^{e}\right)-\mu_{2}} k_{1}-k_{2}-\frac{\zeta_{1}}{\left(1-\zeta_{1}\right)} I\right] \pi_{2}}{\left(\pi_{2}^{*}\right)^{\zeta_{1}}\left(\zeta_{1}-\zeta_{2}\right)} .
\end{aligned}
$$




\section{References}

Abel, A. B., and O. J. Blanchard (1986): "The Present Value of Profits and Cyclical Movements in Investment," Econometrica, 54(2), 249-274.

Bernanke, B., And K. Kuttner (2005): "What Explains the Stock Market's Reaction to Federal Reserve Policy?," Journal of Finance, 60(3), 1221-1257.

Bielecki, T. R., and M. Rutkowski (2004): "Modeling of the Defaultable Term Structure: Conditionally Markov Approach," IEEE Transactions on Automatic Control, 49(3), 361-373.

Bollen, N. (1998): "Valuing Options in Regime-Switching Models," Journal of Derivatives, 6(1), 38-49.

Bonomo, M., and R. Garcia (1996): "Consumption and Equilibrium Asset Pricing : An Empirical Assessment," Jounal of Emprical Finance, 3(3), 239-265.

Buffington, J., and R. Elliott (2002): "American options with regime switching," International Journal of Theoretical and Applied Finance, 5(5), 497-514.

Cagetti, M., L. P. Hansen, T. Sargent, and N. Williams (2002): "Robustness and Pricing with Uncertain Growth," Review of Financial Studies, 15(2), $363-404$.

Campbell, J. Y., and J. Cochrane (1999): "Force of Habit: A ConsumptionBased Explanation of Aggregate Stock Market Behavior," Journal of Political Economy, 107(2), 205-251.

Cecchetti, S. G., P.-S. Lam, and N. C. Mark (1990): "Mean Reversion in Equilibrium Asset Prices, pp. 398-418," American Economic Review, 80(4), 398418. 
Cochrane, J. H. (2005): Asset Pricing. Princeton University Press, Princeton and Oxford, revised edn.

Dai, Q., And K. Singleton (2003): "Term Structure Dynamics in Theory and Reality," Review of Financial Studies, 16(3), 631-678.

Dixit, A., AND R. S. Pindyck (1994): Investment Under Uncertainty. Princeton University Press, Princeton, New Jersey.

Driffill, J., M. Raybaudi, and M. Sola (2003): "Investment Under Uncertainty with Stochastically Switching Profit Streams: Entry and Exit over the Business Cycle," Studies in Nonlinear Dynamics and Econometrics, 7(1), 1-38.

Driffill, J., And M. Sola (1998): "Intrinsic Bubbles and Regime Switching," Journal of Monetary Economics, 42(2), 357-373.

Elliot, R. J., L. Aggoun, And J. B. Moore (1995): Hidden Markov Models: Estimation and Control. Springer-Verlag, New York.

Elliott, R. J., H. Miao, and J. Yu (2007): "General Equilibrium Asset Pricing Under Regime Switching," SSRN: http://ssrn.com/abstract=970382.

— (2009): "Investment Timing under Regime Switching," International Journal of Theoretical and Applied Finance., forthcoming.

Guo, X. (2001): “An Explicit Solution to an Optimal Stopping Problem with Regime Switching," Journal of Applied Probability, 38(2), 464-481.

Guo, X., J. Miao, And E. Morellec (2005): "Irreversible Investment with Regime Shifts," Journal of Economic Theory, 122(1), 37-59.

Hamilton, J. D. (1989): "A New Approach to the Economic Analysis of Nonstationary Time Series and the Business Cycle," Econometrica, 57(2), 357-384. 
Hassett, K., and G. E. Metcalf (1999): "Investment with Uncertain Tax Policy: Does Random Tax Policy Discourage Investment," Economic Journal, 109(457), $372-393$.

Landen, C. (2000): "Bond Pricing in a Hidden Markov Model of the Short Rate," Finance and Stochastics, 4(4), 371-390.

McDonald, R., And D. Siegel (1986): "The Value of Waiting to Invest," Quarterly Journal of Economics, 101(4), 707-727.

Merton, R. C. (1976): "Option Pricing When the Underlying Stock Returns are Discontinuous," Journal of Financial Economics, 4(1-2), 125-144.

NAIK, V. (1993): "Option Valuation and Hedging Strategies with Jumps in the Volatility of Asset Returns," Journal of Finance, 48(5), 1969-1984.

Schwartz, E., And L. Trigeorgis (eds.) (2001): Real Options and Investment under Uncertainty Classical Readings and Recent Contributions. MIT Press, Cambridge, Massachusetts.

Shackleton, M., and R. Wojakowski (2002): "The Expected Return and Exercise Time of Merton-Style Real Options," Journal of Business Finance $E$ E Accounting, 29(3\&4), 541-555.

Veronesi, P. (1999): "Stock market overreaction to bad news in good times: A rational expectations equilibrium model," Review of Financial Studies, 12(5), 9761007.

Wu, S., And Y. Zeng (2005): "A General Equilibrium Model of the Term Structure of Interest Rates under Regime-switching Risk," International Journal of Theoretical and Applied Finance, 8(7), 839-869. 
- (2006): "The Term Structure of Interest Rates under Regime Shifts and Jumps," Economics Letters, 93(2), 215-221.

Yin, G., And Q. Zhang (1998): Continuous-time Markov chains and applications. A singular perturbation approach. Springer, Berlin. 